\title{
Pneumococcal immunization program in Ontario
}

\author{
Monika Naus MD MHSc FRCPC FACPM
}

$I_{a}^{n}$ the early 1990s, under the Mandatory Health Programs and Services Guidelines, Ontario had a stated goal of reducing mortality from pneumonia and influenza. Boards of health in the province were required to promote the use of pneumococcal vaccine among those for whom it was recommended. This created a situation where this was the only vaccine that public health staff were to promote, but not provide. At that time, and today, disease due to invasive Streptococcus pneumoniae is not reportable with the exception of meningitis.

Vaccine was available through the Ontario Drug Benefit Plan to residents of nursing homes and homes of the aged, and to other Ontario Drug Benefit recipients, namely those on social assistance. Through this mechanism, about 1200 doses were distributed in 1991. Pressure on the public health system to incorporate pneumococcal vaccine into the immunization program increased in 1991 when pneumococcal vaccine was removed from the nonformulary list based on a Drug Quality Therapeutics Committee review. It ceased to be available through the Ontario Drug Benefit program except through a section 8 proviso, which was administratively cumbersome. A one-on-one review was required, and the charging of the dispensing fee was allowed. As a result of the outcry that followed this change, the Ontario Drug Benefit program offered to transfer $\$ 1,000,000$ to the public health program. This amount would have been adequate to purchase only 80,000 doses of vaccine per year. Nevertheless, public health pondered a phased approach and began to develop eligibility criteria for a publicly funded pneumococcal program. At this time, vaccine, which was only available from Merck Frosst Canada, was in short supply due to fixed production quotas and an increasing world market. A number of different proposals for limited introduction were discussed internally, but none were satisfactory.
Throughout this time, several other vaccine programs were introduced. In 1989, influenza vaccine became publicly funded. In 1990, oral polio vaccine was introduced, and in 1992 the infant Haemophilus influenza type b program started. In 1993, oral polio vaccine (OPV) was discontinued and inactivated polio vaccines (IPVs) reintroduced. In 1994, the hepatitis B program was expanded to include grade seven students. In 1995, a combined diphtheria-pertussis-tetanus (DPT), IPV, and Haemophilus influenzae type b vaccine was introduced. In 1996, three large expansions of the immunization program occurred including a measles campaign and introduction of a second dose of measles-mumps-rubella (MMR) vaccine, introduction of a publicly funded pneumococcal program for high risk groups and the hepatitis B high school 'catch-up' program. These major initiatives followed the report of the Chief Medical Officer of Health on immunization (1), which contained recommendations in each of these three areas and a recommendation to improve influenza vaccine coverage. The report, which reiterated the content of recent publications showing that use of pneumococcal vaccine in high risk groups reduces morbidity and mortality, raised the spectrum of drug resistance and reminded the readership about longstanding expert advisory committee recommendations for its use. It stated "Ontario doctors and the Ontario immunization program have been slow to adopt this vaccine. There is no good reason for this delay".

In planning for the introduction of the pneumococcal program, the staff of the Ontario Ministry of Health conducted a physician survey in the spring of 1996 to provide information on likely uptake and support required to make optimal use of the vaccine. The survey was conducted by a mailed, selfadministered questionnaire that consisted of nine questions. Surveys were sent to a random sample of 1002 physicians, which constituted $9 \%$ of family and general practitioners in 
the province. A 63\% response rate was obtained. Results were as follows. Physicians were asked whether they routinely recommend the vaccine. Twenty-two per cent stated that they recommend the vaccine routinely to patients over 65 years of age, but only $8 \%$ routinely recommend the vaccine to all risk groups identified by the National Advisory Committee on Immunization (NACI). With respect to barriers to use, 54\% cited lack of awareness of vaccine and the disease, $45 \%$ stated the cost of the vaccine was a problem and $43 \%$ stated that the inconvenient availability presented problems of access. Physicians were asked to estimate their influenza vaccine coverage rate among patients in their practice. Seventy per cent of physicians stated that they immunize more than $60 \%$ of high risk patients, and $80 \%$ of these physicians estimated a similar coverage to be achievable within the first year of a pneumococcal program. Ninety per cent of physicians stated that they would administer the vaccine at the same time as influenza vaccine. Fifty per cent of physicians had no form of recall system to bring high risk patients actively into their office for immunizations. When asked about types of assistance that would be useful to implement the program, $87 \%$ stated that the mass media would be useful and $70 \%$ identified information for health care providers as valuable.

The proposal for implementation included a three-year catch-up program with phased immunization of high risk individuals over a three-year period beginning in 1996/1997 (fiscal year April 1 to March 31, but largely implemented in autumn of each year with the influenza vaccine program), with 400,000 doses distributed in the first year and 500,000 doses distributed in each of the second and third years of the program. Thereafter, beginning in the fiscal year 1999/2000, it was estimated that approximately 80,000 doses per year would be distributed for those turning 65 years of age and entering high risk groups.

The results of the physician surveys were used in planning various components of the program. The program is largely a physician-administered program, with most immunizations given by family doctors and general practitioners. The purchase of the vaccine in the first year of the program was limited by manufacturing capacity from the sole source supplier. The maximum achievable coverage was estimated to be $70 \%$ to $80 \%$ of the target population based on the results of the physician survey, of pilot demonstration projects in the United States and on influenza vaccine distribution in Ontario. A Pneumococcal Program Implementation Working Group was convened, with representation from a number of disciplines and agencies, and met on two occasions to plan various components of the program. The group recommended that, in the first year of the program, residents of long term care (LTC) facilities be targeted, encompassing 56,000 beds with a known coverage averaging $38 \%$ based on previous surveys, but ranging from $0 \%$ to $80 \%$ by region and facility. It was felt that while there may be little potential benefits in this group, the numbers were small and it was a highly accessible population. The other major target group in the first year of the program was persons of any age with underlying medical conditions who were at high risk of disease, and morbidity and mortality from disease. It was decided that healthy seniors could be deferred to later years of the program.

A direct mailing to announce the program to health care providers was carried out in a separate envelope from that used for the influenza program. Product identification stickers accompanied the vaccine for use the personal immunization record and medical chart. A product leaflet was to be included with the mailing materials if this could be revised before the date of the mailing; however, it had not been revised and was therefore not used because of outdated information about revaccination recommendations and administration in conjunction with influenza vaccine. Posters were felt to be very important in prior promotion of influenza vaccine, and a poster was developed for the pneumococcal program, listing high risk groups in large, easy to read lettering. Vaccine was allocated to health units on a per capita basis, with each health unit allocated a maximum ceiling. In turn, physicians were allocated vaccine based on a percentage of their prior year's influenza vaccine order. Physicians were advised to target about one-third of their high risk patient population in each of the three years of the program.

Issues that were identified as especially relevant to residents of LTC facilities and seniors were the use of explicit graphics on promotional materials; joining the promotion of the program to the autumn months in conjunction with influenza vaccine; improving the recording of data on immunization records in order to prevent second dose administration; support for a central immunization registry; development of a model policy for LTC facilities including a plan of treatment consisting of advance consent upon admission to a series of immunizations against pneumococcal disease, influenza, tetanus, diphtheria and Mantoux skin testing; the understanding that written consent was not required; and the understanding that a physician was not required on site during immunization.

It was also recognized that certain risk groups posed a challenge in terms of access to vaccine, including those in retirement homes, patients in chronic care wards of hospitals, community-based seniors and those under 65 years of age with high risk medical conditions. For this latter group, it was recommended that a second year pilot demonstration project be carried out to facilitate targeting of this group in subsequent years of the program. This was felt to be particularly important in light of the changing health care climate in Ontario, as well as hospital closures and decreased resources, which the working group felt did not make immunizations in emergency rooms and before discharge of in-patients a feasible strategy.

The information package mailed to physicians contained a 'dear doctor' letter, which included information about the estimated impact of using the vaccine by reducing the overall incidence of the disease by $60 \%$, the findings that the vaccine is cost effective even when the response is immunologically suboptimal and reference to a recent publication in which 500 life-saving interventions were reviewed, showing that for those over 65 years of age, pneumococcal vaccine costs US $\$ 2,000$ per year of life saved, which compares well with 
other health care interventions $(2,3)$. The package also contained a vaccine order form as well as an order form for fact sheets about the vaccine for patients. Questions and answers about the vaccine and the program were also enclosed, which highlighted the results of an active surveillance program in Metropolitan Toronto that had demonstrated that the $\mathrm{NACI}$ risk groups accounted for $81 \%$ of cases and $94 \%$ of deaths due to this disease (personal communication). Two scientific publications were also included, an article by Fedson (3) about influenza and pneumococcal vaccination of the elderly, which addressed the assumption that pneumonia is the "old man's friend", and an article by Honkanen et al (4), which examined adverse vaccine reaction rates in the elderly following the administration of influenza vaccine alone and concurrently with pneumococcal vaccine. The mailing also contained a promotional poster.

In the first and second year of the programs, pneumococcal vaccine distribution in the province correlated very closely with influenza vaccine distribution, with the majority of doses distributed from September through November, and very little vaccine available thereafter. In the first year of the program, the per capita uptake per health unit for the targeted population ranged from $7 \%$ to $19 \%$, with an average of $17 \%$.

Adverse vaccine reaction monitoring has consisted of routine reporting through the local to provincial health department passive surveillance system. This system is known to be hampered by under-reporting as demonstrated by a Finnish study, which found a rate of 12 to 24 local disabling reactions per $1000 \mathrm{im}$ munizations (4). In contrast, in the first year of the pneumococcal program in Ontario during which 400,000 doses were distributed, a total of 12 adverse events were reported through the passive system. This constituted a rate of three events per 100,000 doses distributed. Six local reactions with severe pain and/or swelling at the injection site were reported, including one with rashes and another with a fever 39 to $40.4^{\circ} \mathrm{C}$. One reaction of arthralgia/arthritis was reported affecting a single joint in an elderly man taking allopurinol for gout. Three reactions in the "miscellaneous - other severe or unusual event" category were reported. These included an eight-year-old child with leukemia with cellulitis at the injection site compatible with a streptococcal infection, an 84-year-old individual who underwent cholecystectomy and died within days following immunization, and a 77-year-old individual who experienced hives and swelling of the mouth for 14 days following immunization, which had been given concurrently with the influenza vaccine; he had experienced a similar reaction following influenza vaccination in 1992. In the second year of the program, which has just begun and during which 500,000 doses of the vaccine will be distributed, only one reaction has been reported to date: a case of anaphylaxis in a 16-year-old asthmatic with a history of milk allergy.

A number of pilot projects to target high risk groups were underway in the second year of the program during the fall of
1997. These projects occurred in two settings. In primary care physicians' offices, the intervention consists of a modification of the patient-management software to allow the production of a list of high risk patients. These patients can then be targeted appropriately for recall to administer the vaccine. In acute care hospital settings, the intervention consists of identification of high risk patients during their hospitalization and the forwarding of a letter to their family physician following discharge in which the physician is provided with information about the benefits of pneumococcal vaccine and the risk factors identified in the newly discharged patient. These pilot projects are in progress and will be evaluated before provincewide implementation.

There are a number of evaluation needs for the program. The effectiveness of the program in reducing morbidity and mortality from invasive Streptococcus pneumoniae disease needs to be evaluated, and the staff who work in the immunization program expect that the results of the active surveillance program, which is ongoing in Metropolitan Toronto and Peel Region, will provide this information. The impact of the program on pneumonia and influenza mortality can be evaluated using registrar general data. Similarly, Ministry of Health staff hope to evaluate impact on hospitalizations using Canadian Institute for Health Information (CIHI) hospital separation data. Coverage of the program also needs to be established and, to date, has only been estimated using vaccine distribution data. Baseline coverage data exist from previous Health Canada surveys such as the general social survey. We hope that such surveys will be repeated on a national basis and are also examining opportunities to collect coverage data specific billing codes for vaccines administered by physicians. Future issues include the uptake of the vaccine program in the third year and ongoing maintenance needs for quantities distributed for subsequent years of the program. Recommendations for booster doses may change in the future, and this will have impact on vaccine requirements. Ontario Ministry of Health staff may need to look more closely at the occurrence of adverse vaccine events following the second and third doses of the vaccine. Finally, introduction of a conjugate pneumococcal vaccine will have implications for program expansions, particularly for children.

\section{REFERENCES}

1. Chief Medical Officer of Health. Immunization - The Next Steps Report of the Chief Medical Officer of Health. Queen's Printer for Ontario, 1995

2. Tengs TO, Adams ME, Pliskin JS, et al. Five hundred life-saving interventions and their cost effectiveness. Risk Anal 1995; 15:369-90.

3. Fedson DS. Influenza and pneumococcal vaccination of the elderly: newer vaccines and prospects for clinical benefits at the margin. Preventive Medicine 1994:23:751-5.

4. Honkanen PO, Keistinen T, Kivela SL. Reactions following administration of influenza vaccine alone or with pneumococcal vaccine to the elderly. Arch Intern Med 1996;156:205-8. 


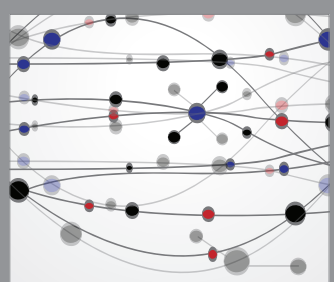

The Scientific World Journal
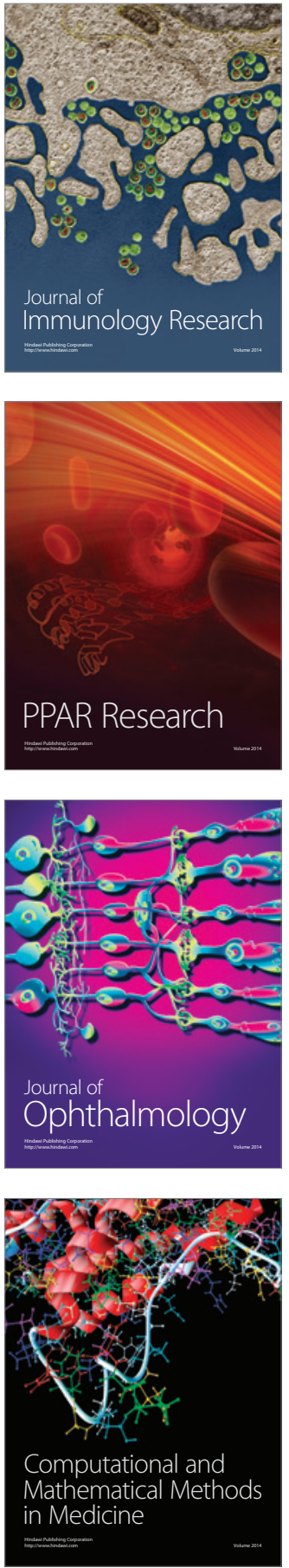

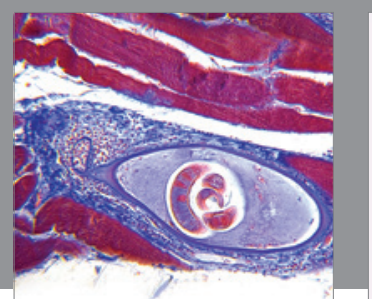

Gastroenterology Research and Practice

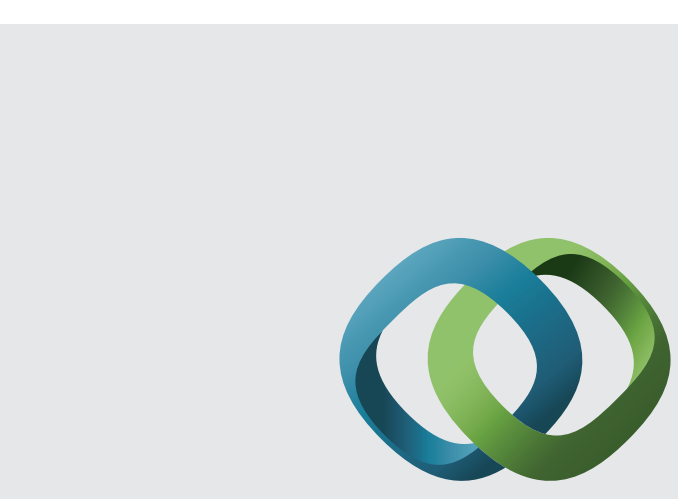

\section{Hindawi}

Submit your manuscripts at

http://www.hindawi.com
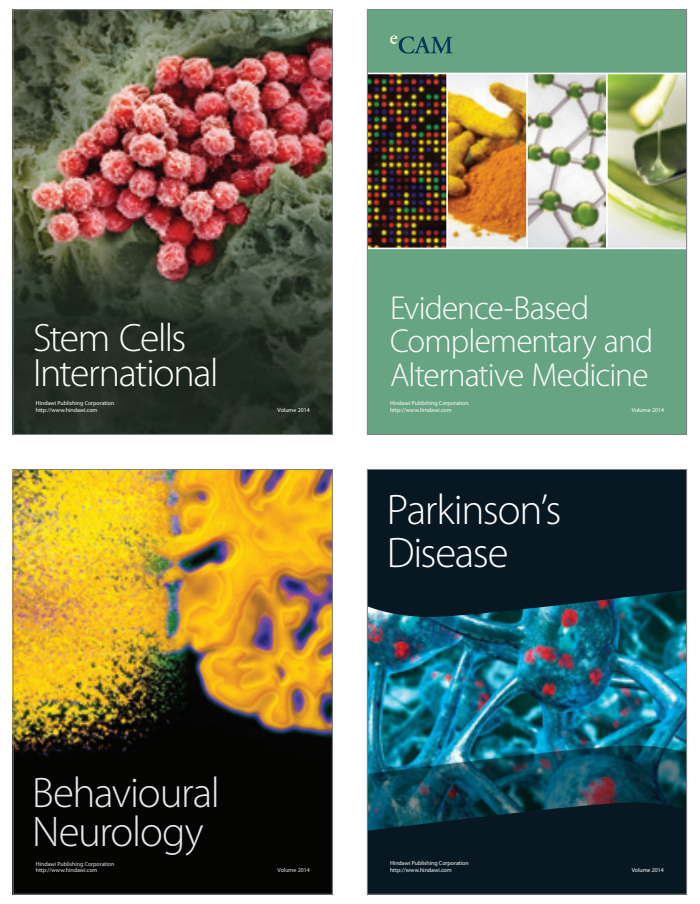
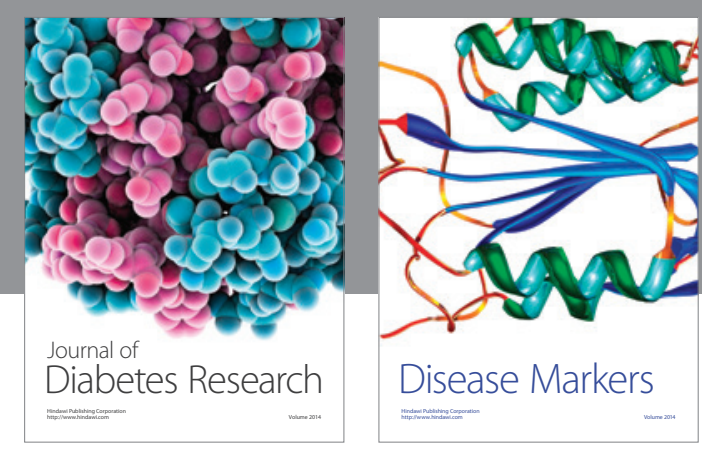

Disease Markers
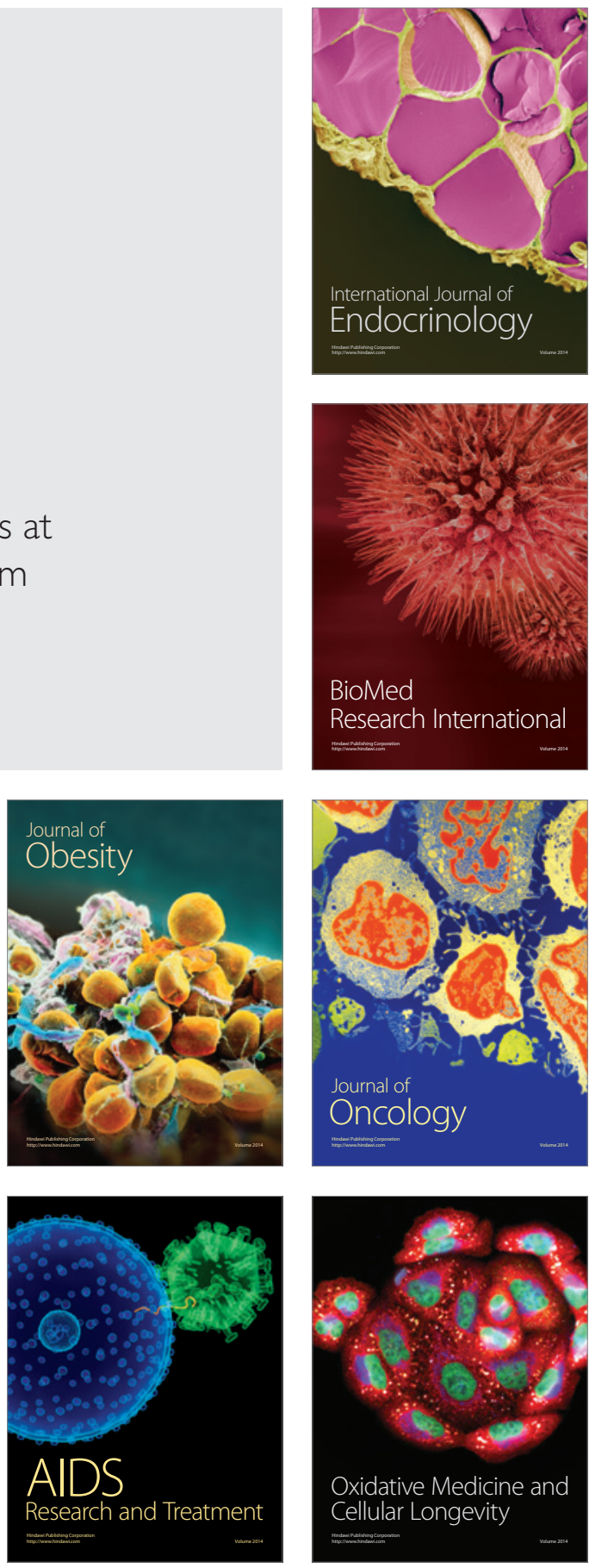